\title{
Empleo de Socrative para la evaluación del trabajo previo en asignaturas prácticas de laboratorio
}

\section{Francesc A. Esteve-Turrillas ${ }^{a}$, Sergio Armenta Estrela ${ }^{a}$ y José Manuel Herrero ${ }^{a}$}

${ }^{a}$ Departamento de Química Analítica, Universitat de València, c/ Dr. Moliner 50, 46100

Burjassot, España (francesc.a.esteve@uv.es, sergio.armenta@uv.es, jose.m.herrero@uv.es)

\begin{abstract}
University students of experimental sciences degrees usually go to the laboratory of practices without having prepared the corresponding activity. This fact leads to a poor preparation of them translated into an effective loss of time available for experimentation, and a reduced acquisition of knowledge and skills, among other aspects. In order to solve this problem, in the present communication, the use of on-line questionnaires using the Socrative platform is described to be used as a prior evaluation system to access the laboratory. The results obtained show that the proposed didactic strategy significantly improves the preparation of the students upon arrival to the laboratory, as well as it provides a high acceptance and satisfaction by the students.
\end{abstract}

Keywords: Socrative, evaluation, laboratory practices, analytical chemistry, on-line test

\begin{abstract}
Resumen
Los estudiantes universitarios de ciencias experimentales suelen acudir a los laboratorios de prácticas sin haberse preparado dicha actividad. Este hecho conlleva a un pobre aprovechamiento de las mismas traduciéndose en una pérdida efectiva del tiempo disponible para la experimentación, y una merma en la adquisición de conocimientos y habilidades, entre otros aspectos. Con objeto de solucionar esta problemática, en la presente comunicación, se describe una experiencia consistente en el empleo de cuestionarios on-line mediante la plataforma Socrative para ser utilizados como sistema de evaluación previo para poder acceder al laboratorio. Los resultados obtenidos muestran que la estrategia didáctica propuesta hizo que la preparación de los estudiantes al llegar al laboratorio mejorara significativamente, así como la elevada aceptación y satisfacción entre el alumnado por la adopción de este tipo de herramientas.
\end{abstract}

Palabras clave: Socrative, evaluación, prácticas de laboratorio, química analítica, examen on-line

\section{Introducción}


En la última década, la implementación del Espacio Europeo de Educación Superior ha supuesto un cambio en el modelo formativo de la educación superior en Europa (de Miguel Díaz, 2013). Este proceso de convergencia ha supuesto inducir al uso en las aulas universitarias de métodos de enseñanza-aprendizaje de tipo activo ya que éstos, teóricamente, pretenden convertir al estudiante en el agente protagonista de su proceso de aprendizaje. Sin embargo, los estudiantes del sistema universitario español están demasiado acostumbrados a ser espectadores en este proceso. En este sentido, concretando en el desarrollo de prácticas de laboratorio de asignaturas de Grados de ciencias experimentales, habitualmente, los estudiantes suelen disponer de un tiempo establecido para poder planificar y llevar a cabo la realización de la práctica (preparación de muestra, diseño de experimentos y medidas experimentales, análisis de resultados, etc.), poder consultar en dicho proceso a los profesores, repetir alguna medida o determinación necesaria, etc. Como paso previo a este proceso, se trabaja con el supuesto de que los estudiantes preparan la sesión de prácticas antes de ir al laboratorio, utilizando el material (guiones detallados de todas las prácticas, material audiovisual de soporte, etc.) previamente disponible en el aula virtual de la asignatura. Sin embargo, se constata que un número importante de los estudiantes se presentan al laboratorio de prácticas sin haber consultado dicho material y sin saber exactamente qué hacer. Este hecho implica una deficiencia en la adquisición de conocimientos/habilidades necesarios para el desempeño correcto de la práctica, junto con una pérdida efectiva del tiempo disponible para la experimentación y un aprovechamiento de la actividad inferior a lo deseable.

Actualmente las tecnologías de la información y la comunicación (TIC) ofrecen enormes posibilidades educativas y los estudiantes presentan un alto grado de dominio de las mismas, ya que viven en una combinación de espacios digitales y reales. Por otro lado, el auge en las tecnologías móviles ha propiciado el desarrollo del denominado aprendizaje móvil (Trillo, 2015), que permite diseñar escenarios de aprendizaje fluidos donde profesores y estudiantes interactúan sin limitaciones espacio-temporales. En este sentido, el empleo de técnicas de gamificación está extendido para su uso como herramienta de aprendizaje interactivo y también para la evaluación del conocimiento (Dicheva et al., 2016). Un formato comúnmente utilizado para convertir los contenidos a revisar o evaluar es mediante el uso de cuestionarios on-line de respuesta inmediata, que permiten crear y aplicar cuestionarios on-line para que los estudiantes respondan en tiempo real mediante sus dispositivos móviles. Actualmente existen aplicaciones web para tal efecto como Google forms, Kahoot, Quizizz, y Socrative, entre otras.

Socrative es una herramienta online (https://www.socrative.com/) que permite al profesor realizar preguntas en clase y a los estudiantes responderlas en el aula en tiempo real a través de cualquier dispositivo móvil (Subirats, 2018). Se trata de una herramienta muy intuitiva, gráfica y que facilita una rápida familiarización. Existen dos versiones de la misma, una gratuita y otra de pago, con más posibilidades. En nuestro caso, se optó por la primera, ya que las opciones que presenta son más que suficientes para la consecución de los objetivos propuestos. Para poder hacer uso de esta herramienta, el profesor accede a la web, donde se registra, y crea su perfil para acceder posteriormente mediante Teacher Login. Una vez 
registrado, el docente crea las cuestiones o tests que considere oportunos, con el número de preguntas que considere apropiado. En la Figura 1 se muestra el menú principal del aula del profesor, así como las distintas posibilidades para diseñar o formular preguntas.

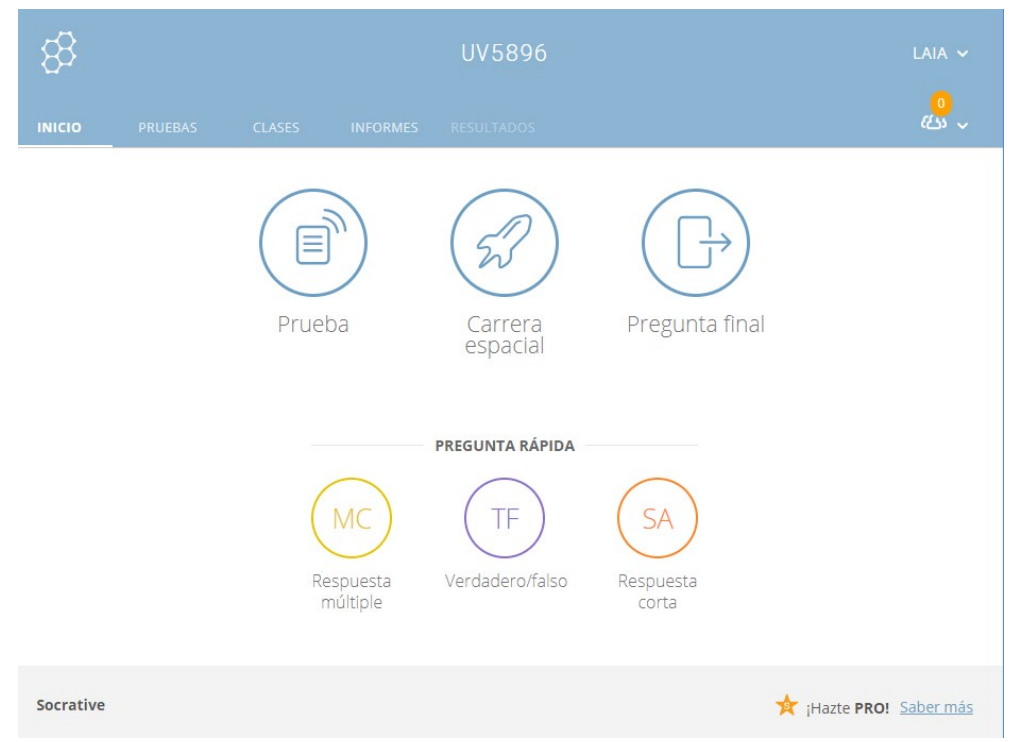

Fig. 1 Menú principal para diseñar cuestiones en Socrative.

Más concretamente, en el menú Pruebas podemos crear cuestionarios con distintos formatos: verdadero/falso, respuesta múltiple y respuesta corta. En nuestro caso, se utilizaron los cuestionarios de respuesta múltiple, y cada cuestionario contenía cinco preguntas, las cuales pueden mostrarse de forma aleatoria a los estudiantes. Asimismo, las preguntas formuladas, preparadas por el equipo docente de la asignatura, contemplaron todos los aspectos de la práctica explicados en el guion correspondiente, desde las bases conceptuales de la experiencia a desarrollar hasta aspectos básicos de las técnicas instrumentales a emplear.

Por su parte, para contestar a estos tests, los estudiantes, desde su dispositivo móvil, deben entrar en el aula del docente (Room number), primero accediendo a Socrative e identificándose como estudiante (Student Login) e introduciendo después el código del aula del docente. A continuación, tras identificarse correctamente, responden desde su dispositivo a las preguntas que se les van formulando. Al terminar el test, cada alumno recibe una puntuación dependiendo de los aciertos obtenidos. Si el número de respuestas acertadas por el estudiante es superior a 3 (sobre 5), se le permite el acceso a la sesión de prácticas. En caso contrario, éste no podrá realizar la práctica, salvo en los casos donde la práctica comprenda dos sesiones, debiéndose de nuevo el alumno hacer el test para poder realizar la misma en la segunda sesión. Además, los resultados de los cuestionarios quedan grabados de forma que el docente pueda descargarlos y recopilar información de cara a la evaluación del trabajo previo.

\section{Objetivos}


El objetivo principal de esta comunicación consiste en el empleo de la herramienta Socrative para realizar test previos en asignaturas de prácticas de laboratorio vinculadas al área de Química Analítica. La metodología propuesta se ha empleado en asignaturas del Grado en Química y del Master Universitario en Técnicas Experimentales en Química que se imparten en la Universitat de València. A través de la realización de los test previos se persiguen los siguientes objetivos específicos:

- Aumentar el grado de preparación del alumnado de asignaturas prácticas de laboratorio en química.

- Concienciar al alumnado de la importancia de una buena preparación de la práctica antes de la asistencia al laboratorio.

- Mejorar la asimilación de conceptos teórico-prácticos relacionados con la asignatura y con ello mejorar las competencias adquiridas.

- Fomentar el empleo de herramientas y aplicaciones disponibles en Internet para la realización de test rápidos en el aula.

- Evitar que las prácticas de laboratorio se conviertan en la mera aplicación de una "receta de cocina".

\section{Desarrollo de la innovación}

La metodología docente innovadora que se propone, basada en el empleo de la aplicación Socrative, se ha aplicado en dos asignaturas teórico-prácticas de laboratorio que se imparten en el Departamento de Química Analítica de la Universitat de València durante el curso 2018-2019. Los seminarios teóricos de la asignatura se imparten en aulas con una capacidad de 60 personas. Las prácticas de laboratorio se realizan en un laboratorio docente de la Facultad de Química de la Universitat de València, que consta de bancadas de laboratorio, con una capacidad de 48 alumnos, con el material necesario para la preparación de muestras, así como adecuada ventilación, sistemas de extracción, y equipada con los sistemas de seguridad habituales en un laboratorio docente. Adicionalmente, el alumno debe llevar puesto en todo momento los convenientes equipos de protección individual (bata, gafas protectoras y guantes), así como disponer del guion del laboratorio y un cuaderno de trabajo. En un laboratorio adyacente se encuentran los equipos instrumentales donde se realizan las medidas analíticas necesarias.

\subsection{Laboratorio de Análisis Instrumental Aplicado - Grado en Química}

\subsubsection{Contexto de aprendizaje}

La asignatura de Laboratorio de Análisis Instrumental Aplicado es una asignatura de carácter optativo que se imparte en el primer cuatrimestre del cuarto curso del Grado en Química. El número de estudiantes matriculados en el curso 2018-2019 es de 36 alumnos divididos en tres grupos de 12 estudiantes, que se imparten en castellano, valenciano e inglés como lenguas vehiculares. La asignatura incluye 12 horas de clases de teoría y 48 horas de prácticas de laboratorio como clases presenciales de asistencia obligatoria con un 
total de 6 créditos ECTS. La guía docente de la asignatura indica que el alumno debe realizar adicionalmente $13 \mathrm{~h}$ de elaboración de trabajos en grupo, $13 \mathrm{~h}$ de elaboración de trabajos individuales, $23 \mathrm{~h}$ de estudio y trabajo autónomo, $13 \mathrm{~h}$ de lecturas de material complementario, $14 \mathrm{~h}$ de preparación de actividades de evaluación y 14 h de preparación de clases prácticas y de problemas.

Con el fin de abordar con éxito esta asignatura, los estudiantes deben poseer unos conocimientos previos relativos a la forma de trabajo general en un laboratorio de Química Analítica, así como conocimientos sólidos sobre los aspectos teóricos de Química Analítica incluyendo las diversas técnicas instrumentales. Por tanto, resulta recomendable que hayan superado en cursos anteriores las asignaturas de Química Analítica I, Química Analítica II y Química Analítica III así como los dos laboratorios que se cursan en segundo y tercer año del Grado en Química.

\subsubsection{Contenidos de la asignatura}

La descripción de los contenidos de la asignatura Laboratorio de Análisis Instrumental Aplicado incluye la realización de 11 prácticas de laboratorio de análisis químico, con una duración de $3 \mathrm{~h}$ por sesión, distribuidas en cuatro bloques:

\section{Bloque 1. Análisis de muestras medioambientales.}

Práctica 1. Determinación de humedad, $\mathrm{pH}$, conductividad y materia orgánica en suelos (2 sesiones).

Práctica 2. Determinación de fósforo en suelo por formación de azul de fosfomolibdeno (1 sesión).

Práctica 3. Determinación de cadmio asimilable en suelos mediante espectroscopia de absorción atómica con cámara de grafito (1 sesión).

\section{Bloque 2. Análisis de alimentos y productos industriales.}

Práctica 4. Determinación de proteínas en pasta por el método Kjeldahl (2 sesiones).

Práctica 5. Determinación refractométrica del grado de humedad en miel y sólidos solubles en zumo de naranja ( 0.5 sesión).

Práctica 6. Análisis de ácidos grasos en aceites vegetales por cromatografía de gases $(0.5$ sesión).

Práctica 7. Determinación de tocoferoles en aceites vegetales por cromatografía líquida de fase reversa con detección fluorimétrica (2 sesiones).

\section{Bloque 3. Análisis clínico y farmacéutico.}

Práctica 8. Determinación quimioluminiscente de hipoclorito sódico en un producto de limpieza (2 sesiones).

Práctica 9. Determinación cinético-espectrofotométrica de creatinina con picrato alcalino (1 sesión). 
Práctica 10. Determinación de selenio mediante voltametría de redisolución catódica (2 sesiones).

\section{Bloque 4. Búsqueda y selección del método analítico más apropiado.}

Práctica 11a. Determinación de metales en monedas (2 sesiones).

Práctica 11b. Determinación de colorantes alimentarios en gominolas (2 sesiones).

Práctica 11c. Determinación de rivoflavina en orina (2 sesiones).

Cabe indicar que en este último bloque, a cada estudiante se le asigna una única práctica de laboratorio en el que debe hacer una búsqueda bibliográfica previa para obtener el mejor procedimiento experimental para resolver el problema analítico propuesto y llevarlo a cabo en la sesión de laboratorio.

\subsection{Laboratorio de Análisis de Alimentos- Master Universitario en Técnicas Experimentales en Química}

\subsubsection{Contexto de aprendizaje}

La asignatura de Laboratorio de Análisis de Alimentos es una signatura de carácter obligatorio que se imparte en el segundo cuatrimestre del Master Universitario en Técnicas Experimentales en Química. El número de estudiantes matriculados en el curso 2018-2019 es de 27 alumnos divididos en dos grupos de 13 y 14 estudiantes respectivamente, que se imparten utilizando castellano como lengua vehicular. La asignatura incluye 30 horas de prácticas de laboratorio de carácter $100 \%$ presencial, así como $6 \mathrm{~h}$ de elaboración de trabajos individuales, $15 \mathrm{~h}$ de estudio y trabajo autónomo, $6 \mathrm{~h}$ de lecturas de material complementario, $6 \mathrm{~h}$ de preparación de actividades de evaluación, $6 \mathrm{~h}$ de preparación de clases prácticas y de problemas, y $6 \mathrm{~h}$ de resolución de casos prácticos, con un total de 3 créditos ECTS.

El perfil del estudiante del Master Universitario en Técnicas Experimentales en Química corresponde a personas que han finalizado titulaciones previas en el ámbito de las ciencias experimentales como Grado o Licenciatura en Química, Farmacia, Ciencia y Tecnología de Alimentos, Nutrición Humana y Dietética, Bioquímica, Ciencias Ambientales, Ingeniería Química, Tecnología Química, o títulos correspondientes a otras ciencias experimentales y tecnologías afines. El Master Universitario en Técnicas Experimentales en Química presenta una elevada orientación profesional en el ámbito de la química analítica y está dotado de un elevado nivel de experimentalidad, así como formación investigadora. Por lo que se requiere un elevado grado de conocimientos previos sobre química y trabajo experimental en el laboratorio de química.

\subsubsection{Contenidos de la asignatura}

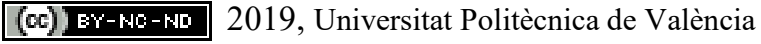


La descripción de los contenidos de la asignatura Laboratorio de Análisis de Alimentos incluye la realización de 4 prácticas de laboratorio, con una duración de 4 h por sesión, orientadas al análisis químico de alimentos por medio de métodos oficiales de análisis o de garantía contrastada.

Práctica 1. Identificación de impurezas y determinación de metanol en bebidas alcohólicas de alta graduación mediante cromatografía de gases ( 2 sesiones).

Práctica 2. Determinación espectrofotométrica de índices K en aceites de oliva (1 sesión).

Práctica 3. Determinación de ocratoxina en vinos por cromatografía líquida de alta resolución con detección fluorimétrica (2 sesiones).

Práctica 4. Evaluación de residuos de plaguicidas en naranjas por cromatografía de gases acoplada con espectrometría de masas (1 sesión).

\subsection{Descripción de la tarea}

El desarrollo de prácticas de laboratorio, tanto en asignaturas de Grado como de Máster, requiere de una preparación previa del alumnado para obtener un rendimiento óptimo del proceso de enseñanza/aprendizaje en las prácticas a realizar. Esta preparación previa incluye tanto el repaso por parte del alumno de conceptos teóricos necesarios para el desarrollo adecuado de la tarea, como aclaraciones puntuales sobre precauciones a tener en cuenta en el desarrollo de ésta. La experiencia previa en la impartición de asignaturas prácticas de laboratorio indica que el alumno no suele realizar el trabajo previo necesario para realizar las prácticas propuestas, lo que conlleva a una mala asimilación de los conceptos teóricos prácticos que se pretenden trabajar y a una adquisición incompleta de las competencias requeridas. Asimismo, esta situación suele provocar frecuentemente retrasos en el desarrollo de la práctica, lo que conlleva a una mala realización de esta. En los últimos años se han propuestos diferentes estrategias para fomentar el trabajo previo de los alumnos en la realización de asignaturas prácticas para revertir esta situación no deseada, como: i) proponer la realización de cuestiones previas relacionadas con la práctica a realizar, ii) realizar seminarios previos con el contenido de la práctica, o iii) proporcionar previamente material audiovisual relacionado. A pesar de que las estrategias comentadas anteriormente han tenido un grado de mejora aceptable, el nivel de conocimientos del alumno resulta aún insuficiente para un rendimiento óptimo del proceso de enseñanza/aprendizaje y una adquisición apropiada de competencias.

En el curso 2018/2019 se ha propuesto la realización de test rápidos que se realizan en los primeros minutos de la sesión de prácticas, que contienen 5 preguntas tipo test con una sola respuesta correcta sobre la práctica a realizar en esa sesión de laboratorio. En la Figura 2 se muestra un ejemplo de cuestionario desarrollado para una práctica determinada. 

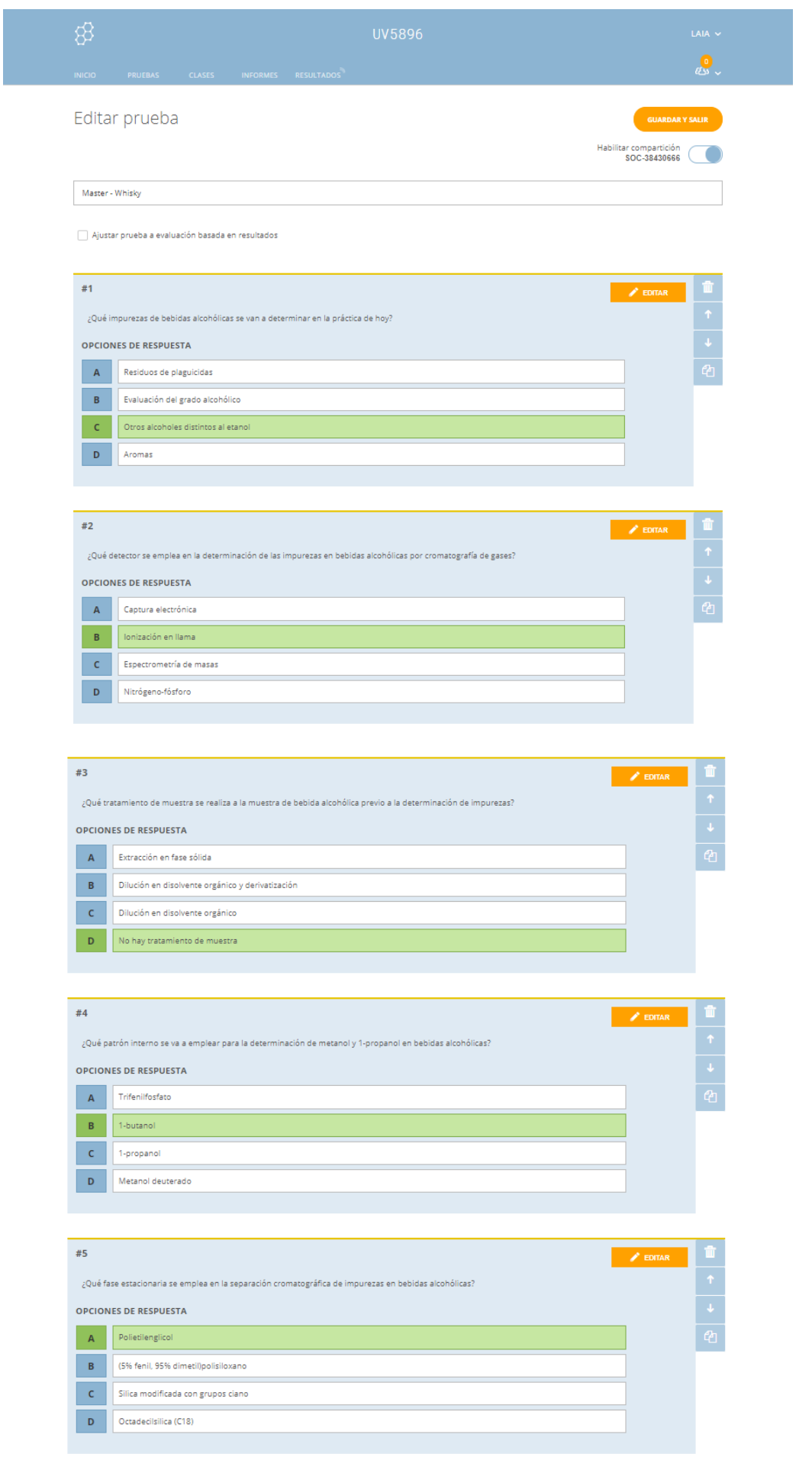

preguntas

+ RESPUESTA MULTIPLE + + VERDADEROFAL SO + + RESPUESTA CORTA

Fig. 2 Ejemplo de Quiz desarrollado usando Socrative.

(cc) EY-NC-ND 2019, Universitat Politècnica de València 
La contribución del test previo a la nota final de la asignatura es de un $10 \%$ para el Laboratorio de Análisis Instrumental Aplicado y de un 20\% para el Laboratorio de Análisis de Alimentos. Adicionalmente, aquellos alumnos que no superen la prueba no podrán asistir a la práctica de ese día, viéndose reflejado en la calificación final de la asignatura. Con el objetivo de agilizar el proceso se ha empleado la aplicación Socrative para la realización de dichos exámenes previos. El empleo de Socrative permite realizar el test en el mismo laboratorio a través del empleo de los teléfonos móviles de los alumnos conectados a la red wifi eduroam, además permite obtener los resultados del test rápidamente en tiempo real, para tomar las decisiones oportunas en caso de no superar la prueba. En el caso de prácticas de dos días de duración se le permite al alumno repetir la prueba el segundo día y en el caso de superarla, realizaría la parte correspondiente a la segunda jornada de prácticas.

Finalmente, en la última sesión de prácticas se realiza un cuestionario anónimo a los alumnos para establecer su grado de satisfacción en la realización de test previos a las prácticas de laboratorio, así como en el empleo de la aplicación Socrative. Asimismo, el cuestionario final contiene preguntas acerca del desarrollo de la asignatura, muy útiles para el profesorado, para identificar qué prácticas han resultado más interesantes para los alumnos, qué técnica analítica les hubiera gustado profundizar con mayor detalle, qué práctica hubieran modificado, o si el desarrollo de la asignatura ha mejorado sus conocimientos teórico-prácticos en el análisis químico. La Tabla 1 muestra las preguntas realizadas en el cuestionario final realizado por los alumnos tanto del Laboratorio de Análisis Instrumental Aplicado, como del Laboratorio de Análisis de Alimentos.

\section{Resultados de la actividad}

El desarrollo de test previos se realizó sin ninguna incidencia por parte de alumnos o los docentes implicados. Desde un punto de vista operativo, la realización de los test previos no dificulta el desarrollo de las prácticas en el horario propuesto, ya que al realizarse de forma autónoma por cada alumno a través de su Smartphone, se puede realizar en apenas 5 minutos. En el momento de finalizar el test el alumno obtiene automáticamente el resultado de la prueba, lo que facilita la agilidad del proceso. Además el docente obtiene, también a través de su Smartphone, el resultado de los test en tiempo real, pudiendo tomar las decisiones oportunas en el mismo momento en que el alumno finaliza su prueba.

Los resultados obtenidos en cada una de las pruebas realizadas para cada práctica de laboratorio se muestran en la Figura 3. Como se observa el resultado obtenido en los tests previos fueron más que satisfactorios, con una nota final de $9.2 \pm 0.6$ para el Laboratorio de Análisis Instrumental Aplicado y de $9.0 \pm 0.6$ para el Laboratorio de Análisis de Alimentos. La mayoría de los estudiantes obtuvieron una calificación entre 8 y 10 para la gran mayoría de las prácticas realizadas. En un porcentaje mucho menor, algunos alumnos obtuvieron una nota de 6 en prácticas concretas, pero en general el resultado global fue excelente para todos ellos. En un único caso, un alumno obtuvo un resultado de 4, por lo que no pudo realizar la práctica correspondiente. 
Tabla 1. Preguntas incluidas en el cuestionario final.

\begin{tabular}{|c|c|c|c|c|}
\hline P1 & \multicolumn{4}{|c|}{ ¿Qué práctica te ha resultado más interesante? } \\
\hline $\mathbf{P 2}$ & \multicolumn{4}{|l|}{ Respuesta abierta } \\
\hline P3 & \multicolumn{4}{|l|}{ Respuesta abierta } \\
\hline \multirow{2}{*}{ P4 } & \multicolumn{4}{|c|}{ ¿Qué práctica/s eliminarías o modificarías? } \\
\hline & \multicolumn{4}{|l|}{ Respuesta abierta } \\
\hline \multirow{2}{*}{ P6 } & \multicolumn{4}{|c|}{ Valora tu experiencia en la asignatura } \\
\hline & Muy positiva & Positiva & Negativa & Muy negativa \\
\hline \multirow{2}{*}{ P7 } & \multicolumn{4}{|c|}{ ¿Recomendarías realizar la asignatura a un compañero? } \\
\hline & Sí & Probablemente sí & Probablemente nc & No \\
\hline P8 & \multicolumn{4}{|c|}{ ¿Cuánto tiempo has dedicado a la preparación previa de cada práctica? } \\
\hline \multirow{2}{*}{ P10 } & \multicolumn{4}{|c|}{ ¿Has empleado Socrative con anterioridad en alguna asignatura? } \\
\hline & Sí, varias veces & Sí, una única vez & Nunca & Creo que no \\
\hline \multirow{2}{*}{ P11 } & \multicolumn{4}{|c|}{ ¿Te ha resultado fácil de utilizar la aplicación Socrative? } \\
\hline & \multicolumn{2}{|c|}{$\mathrm{Si}$} & \multicolumn{2}{|c|}{ No } \\
\hline \multirow{2}{*}{ P12 } & \multicolumn{4}{|c|}{ Valora tu experiencia con el uso de Socrative en esta asignatura. } \\
\hline & Muy positiva & Positiva & Negativa & Muy negativa \\
\hline \multirow{2}{*}{ P13 } & \multicolumn{4}{|c|}{ ¿Emplearías Socrative en alguna otra asignatura? } \\
\hline & Sí & Probablemente sí & Probablemente no & No \\
\hline \multirow{2}{*}{ P14 } & ¿Te gustaría indicar al & nentario adicional? & ón, crítica, valoracio & \\
\hline & Respuesta abierta & & & \\
\hline
\end{tabular}




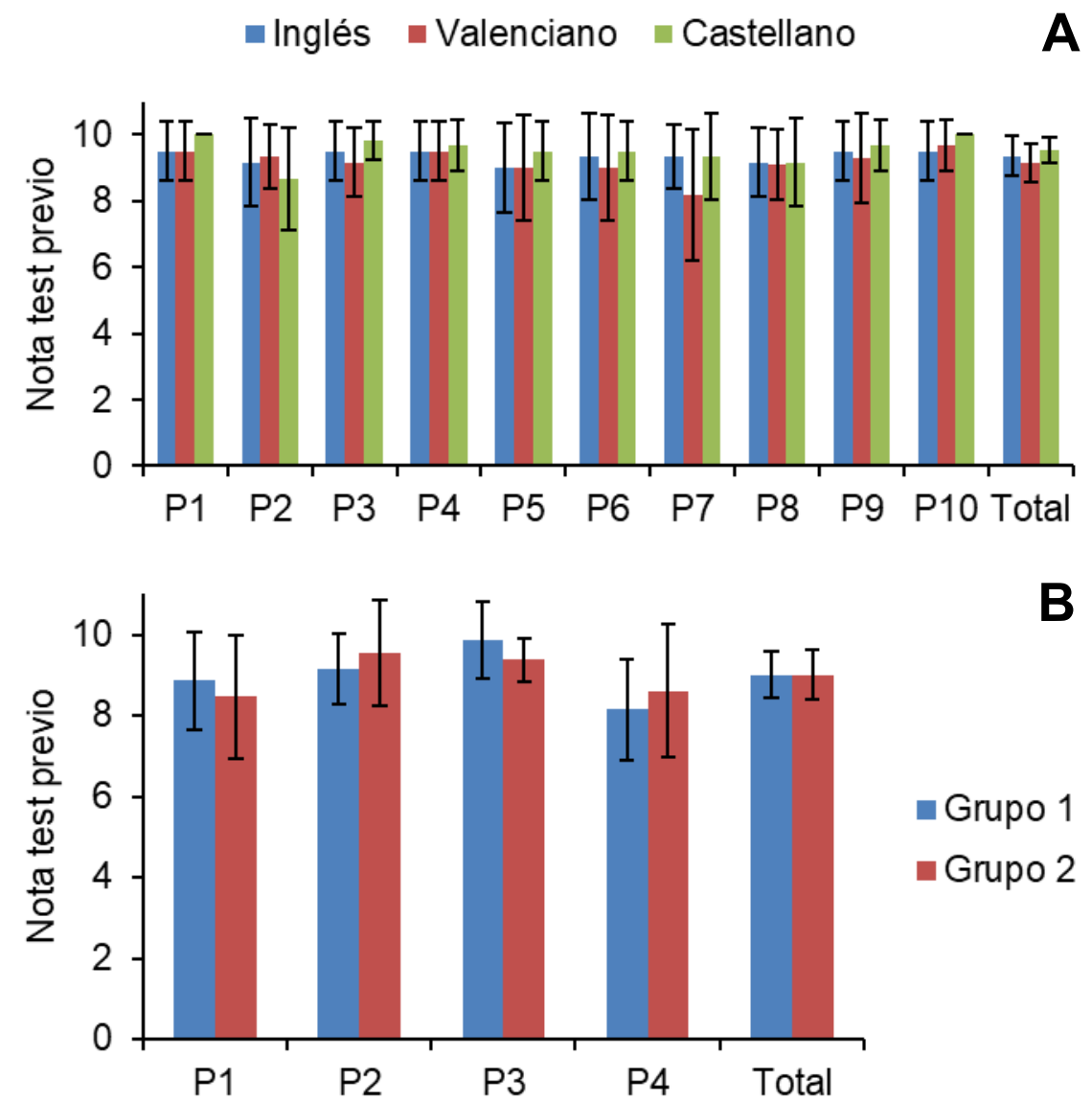

Fig.3 Resultados obtenidos en los test previos a los alumnos del Laboratorio de Análisis Instrumental Aplicado (A) y del Laboratorio de Análisis de Alimentos (B).

Como se ha indicado anteriormente, en la última sesión de prácticas se realiza un cuestionario anónimo a los alumnos para establecer su grado de satisfacción respecto a las prácticas realizadas, indicando qué prácticas les han resultado más interesantes y aquellas que desearían eliminar o modificar en cursos posteriores. Esta información resulta de gran utilidad para el profesorado a la hora de proponer cambios en la programación de las prácticas de esa asignatura para años futuros. La Figura 4 muestras los resultados obtenidos, donde se identifican claramente qué prácticas les han resultado más interesantes y cuáles no. Adicionalmente, al tratarse de una respuesta abierta el alumno puede ampliar el motivo de su respuesta, obteniendo el docente una retroalimentación inmediata de los motivos por los que esa práctica no resulta del agrado del alumno.

En este cuestionario final también se incluyeron preguntas para cuantificar el grado de satisfacción general de la asignatura, así como cuestiones relacionadas con el empleo de tests previos para evaluar el trabajo previo a las prácticas a realizar. La Figura 5 muestras los resultados obtenidos. Como se observa, la asignatura cumplió las expectativas para un $83-85 \%$ de los alumnos y parcialmente para el 17-15\% para las asignaturas de Grado y 
Master, respectivamente. Siendo su experiencia muy positiva o positiva para el 97-100\% de los alumnos y negativa para un 3 y $0 \%$ de los alumnos.

\section{- ¿Qué práctica te ha resultado más interesante? \\ - ¿Qué práctica/s eliminarías o modificarías?}
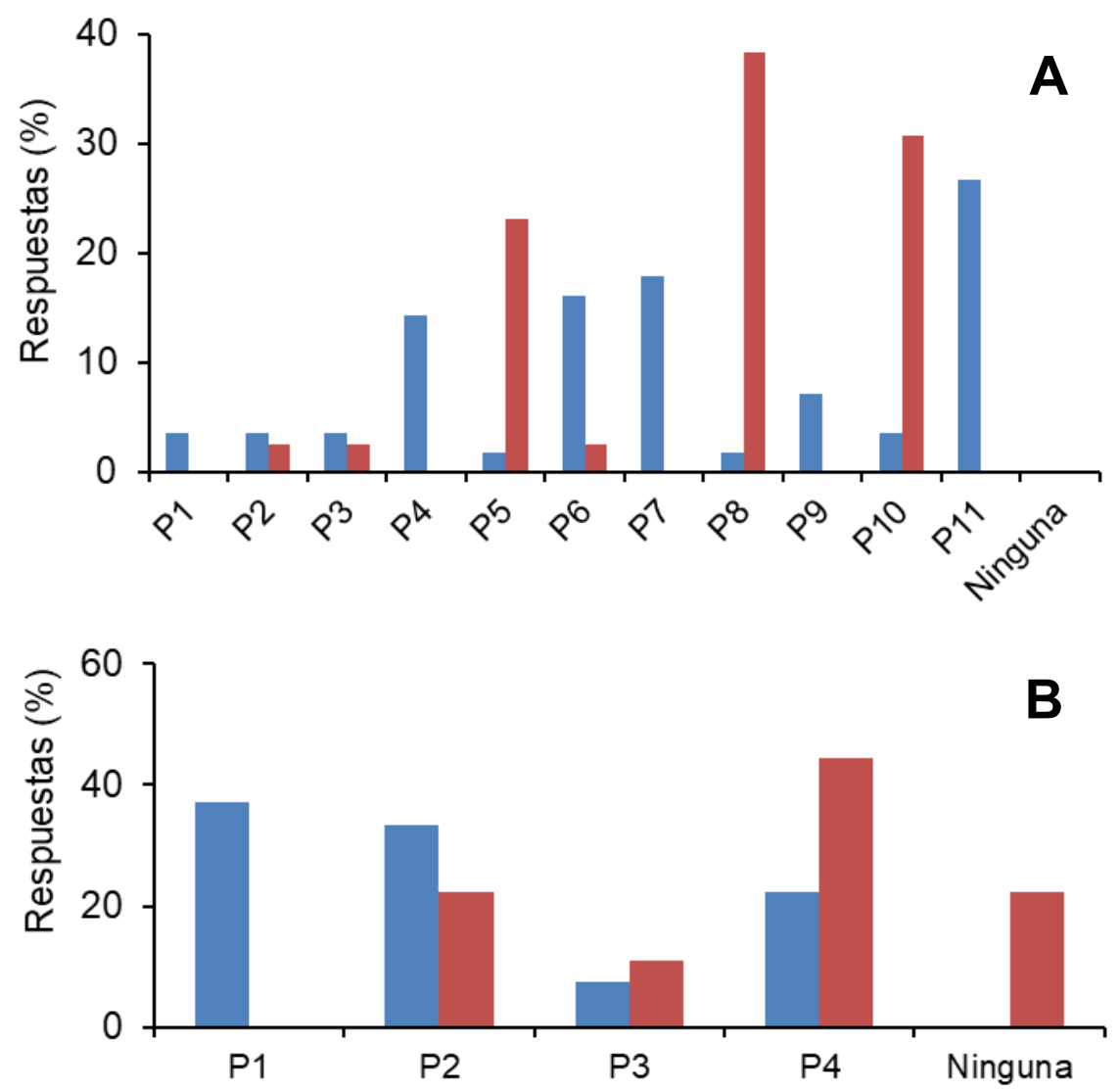

Fig. 4 Valoración de las prácticas realizadas por los alumnos del Laboratorio de Análisis Instrumental Aplicado (A) y del Laboratorio de Análisis de Alimentos (B).

En cuanto al tiempo dedicado a la preparación de las prácticas se observan resultados diferentes para estudiantes de Grado y de Master. En el caso de estudiantes de Grado la gran mayoría (67\%) dedicaron un promedio de 30 a 60 min en la preparación previa de las prácticas, un $22 \%$ de 10 a $20 \mathrm{~min}$, y un $6 \%$ dedicaron o menos de 10 min o más de una hora. En cuanto a estudiantes de Master, el tiempo dedicado a la preparación de las prácticas fue de 10 a 30 min para un $59 \%$ de los alumnos, de 30 a 60 min para el $33 \%$, menos de 10 min para el $4 \%$, y más de una hora para el $4 \%$.

Respecto a su valoración en la realización de test previos a la realización de las prácticas, la gran mayoría de los alumnos tuvo una apreciación entre muy positiva y positiva, siendo de un $88 \%$ para estudiantes de Grado y de un $100 \%$ para estudiantes de Master. Mientras que solo un $11 \%$ de los estudiantes de Grado la valoraron como negativa o muy negativa. 


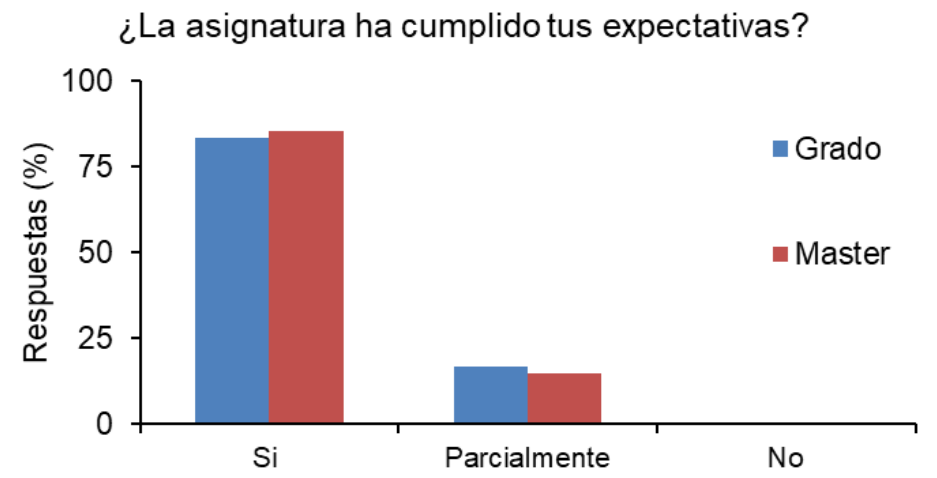

Valora tu experiencia en esta asignatura

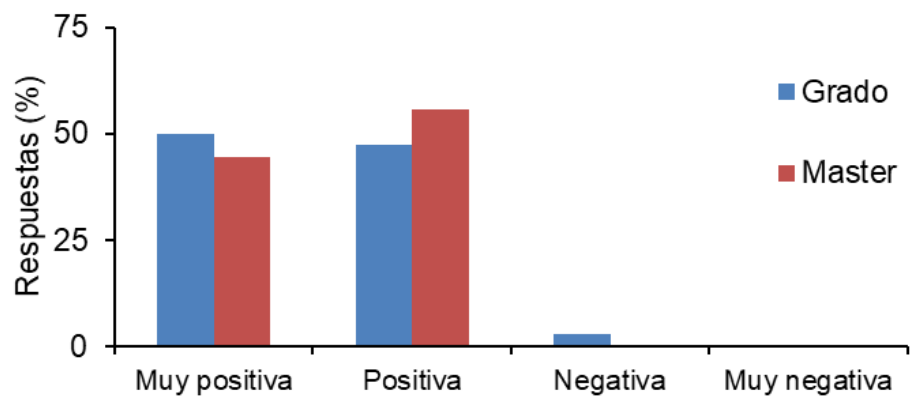

¿Cuánto tiempo has dedicado a la preparación previa de cada práctica?

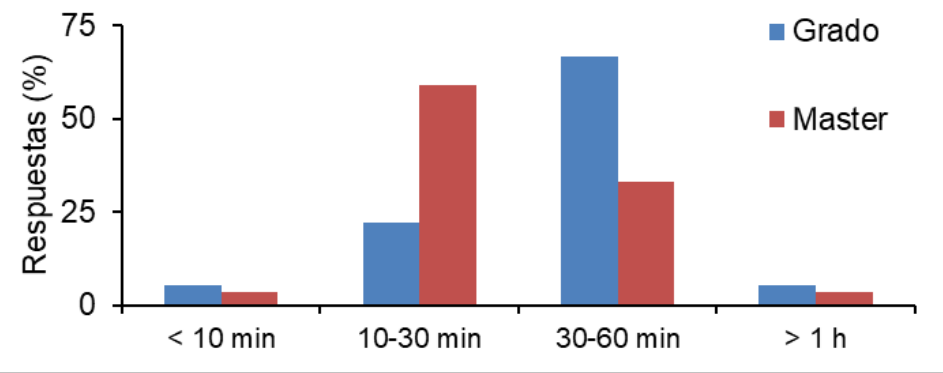

¿Cómo valoras la realización de test previos a la realización de las prácticas?

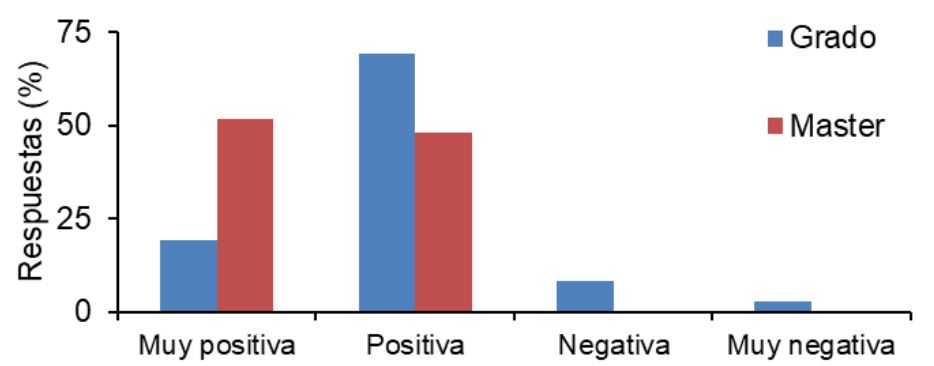

Fig. 5 Valoración de la asignatura y de la realización de cuestionarios previos por los alumnos del Laboratorio de Análisis Instrumental Aplicado del Grado en Química (azul) y del Laboratorio de Análisis de Alimentos del Master Universitario en Técnicas Experimentales en Química (rojo). 
Finalmente, los resultados de la valoración global del empleo de la herramienta Socrative se encuentra en la Figura 6. Un $28 \%$ de los estudiantes de Grado y un $100 \%$ de estudiantes de Master emplearon dicha aplicación en otras asignaturas. Siendo su experiencia mayoritariamente favorable, un 28 y $48 \%$ muy positiva y un 69 y $52 \%$ positiva, para estudiantes de Grado y Master, respectivamente. En cuanto al empleo de Socrative en futuras asignaturas cabe destacar que los resultados fueron más heterogéneos: un $70 \%$ de los estudiantes de Grado y un 90 \% de Master emplearían sí y probablemente sí Socrative en otras asignaturas, mientras que un $30 \%$ de los estudiantes de Grado y un $7 \%$ de Master preferirían no volver a emplearlo.
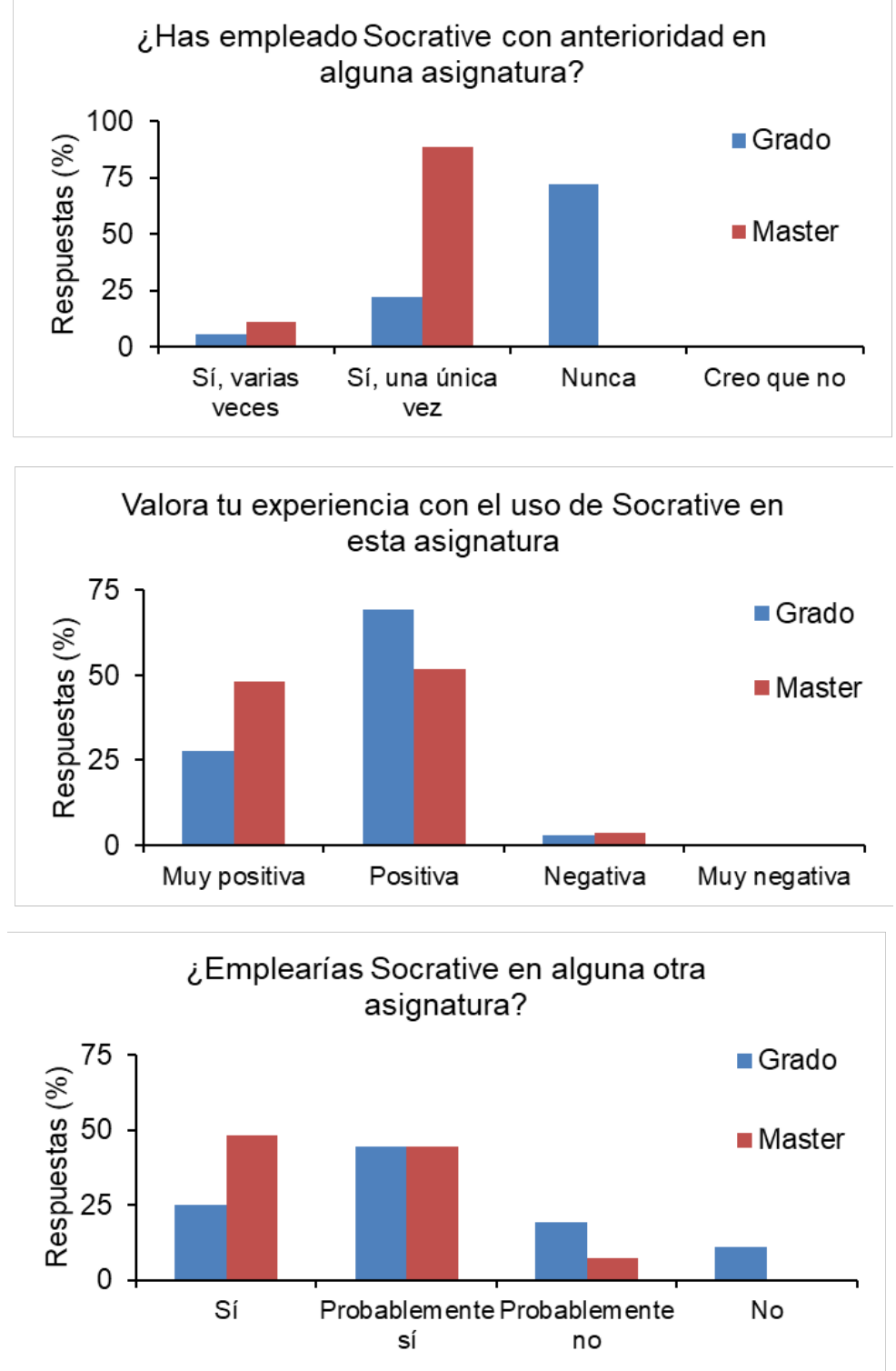

Fig. 6 Valoración del empleo de Socrative por los alumnos del Laboratorio de Análisis Instrumental Aplicado del Grado en Química (azul) y del Laboratorio de Análisis de Alimentos del Master Universitario en Técnicas Experimentales en Química (rojo). 


\section{Conclusiones}

Con la actividad propuesta se ha puesto de manifiesto la eficacia del empleo de herramientas on-line para la realización de evaluaciones ágiles, sencillas y rápidas en el aula o laboratorio. El empleo del Smartphone del alumno en combinación con App o herramientas web abre las posibilidades tanto a la hora de impartir y afianzar conceptos, como a la hora de realizar evaluaciones de las competencias adquiridas. Adicionalmente, dichas herramientas permiten la obtención instantánea de resultados, lo que permite al docente llevar a cabo medidas correctoras o hacer más hincapié en ciertos aspectos a mejorar en el desarrollo de las prácticas de laboratorio propuestas. Los resultados obtenidos son aún preliminares y muestran una leve mejora en la calificación global obtenida por los alumnos en ambas asignaturas respecto al año anterior. En cualquier caso, en años venideros se prevé continuar con el desarrollo de la actividad propuesta para comprobar si existe una mejora en las habilidades desarrolladas por los alumnos con una mayor significancia estadística.

\section{Agradecimientos}

Este trabajo ha sido financiado por la Universitat de València (Vicerectorat d'Ocupació i Programes Formatius) a través del proyecto de innovación docente UV-SFPIE_RMD18190354.

\section{Referencias}

DE MIGUEL DÍAZ, M. (2013) La reforma curricular y metodológica de las enseñanzas universitarias. Una mirada crítica sobre la construcción del Espacio Europeo de Educación Superior, Pulso 36, 13-35.

TRILLO, M.P. (2015). Principios pedagógicos del aprendizaje ubicuo, en Vázquez-Cano, E., Sevillano, M.L. (Eds.), Dispositivos digitales móviles en Educación. El aprendizaje ubicuo, Narcea, Madrid, 39-48.

DICHEVA, D., DICHEV, C., AGRE, G., ANGElOVA, G. (2016) Gamification in education: a systematic mapping study, J. Educ. Technol. Soc. 18, 75-88.

SUBIRATS, X. (2018). Socrative, una herramienta interactiva para el aprendizaje y la evaluación a través del móvil, Actualidad Analítica 63, 7-10. 